\title{
Avoidance of Confusion between Similar looking Characters in Neuro-fuzzy based License Plate Recognition
}

\author{
Choubey Siddhartha, Sinha G.R., IEEE / IACSIT Member, Patel Bagwati Charan, Choubey Abha, \\ Thakur Kavita, IEEE Member
}

\begin{abstract}
This paper presents a new methodology for the image segmentation and character recognition from standard Indian License number plates. Firstly it gets input of the segmented characters that is partitioned by our pixel Clustering partitioning method, in which we eliminate similar part from the character and match it by judging template and return identified character. This partitioning may be applied horizontally or vertically. Decision that the characters are partitioned horizontally or vertically depends on their subgroup. Before sub grouping we have to group the characters on the basis of the number of holes in it and then we subgroup on the basis of some similar features like $\mid, /, 1$, _ , ( , - , etc. If we have alphabet $T$ and $I$ where similar portion is $I$ then both will go to same subgroup and we partition it horizontally. This method eliminates the problem of confusion between similar looking elements like C, G and T, I, 1 , $J$ etc by exploiting the small but important differences among them.
\end{abstract}

Index Terms-Pixel clustering; character segmentation; licence number plate; partitioning technique.

\section{INTRODUCTION}

At present, a vital part in transportation is played by vehicles. In recent years, the usage of vehicles has been increasing on account of population growth and human needs. Hence control of vehicles is becoming a huge problem that is hard to solve [1]

An image processing technology called License Plate Recognition (LPR) that is used to identify vehicles by their license plates is a kind of automatic vehicle identification [2].

Image acquisition, license plate detection, character segmentation and character recognition are four major phases of License Plate Recognition [3-4]. Several areas including traffic volume control, unsupervised park monitoring, traffic law enforcement and auto toll collections on highways extensively use license plate recognition applications [5-6]. In this we introduce a new algorithm for character extraction and recognition based on based on fuzzy logic and analyzing some pattern recognition based works.

Manuscript received April 8, 2011, revised October 7, 2011.

Siddartha Choubey, G.R.Sinha, Bhagwati Charan Patel, Abha Choubey are with Shri Shankaracharya College of Engineering and Technology, Bhilai, India. (e-mail: sidd25876@gmail.com; drgrsinha@ieee.org; bhagwaticharanpatel@gmail.com; abhachoubeyphd@gmail.com).

Kavita Thakur is with Pt. Ravishankar Shukla University, Raipur, India. (e-mail:kavithakur@rediffmail.com)

\section{RELATED WORKS}

W Ping -Wen Ong et al. uses a special space-variant sensor based on complex log mapping to do character recognition in real scenes. This paper solves the problem where the workspace are variant such as some where no space between characters or some where one or more spaces is there and for this purpose it uses a space variant sensor that allow to coverage of a large workspace, but which provide a high resolution area in a small central (foveal) region whose resolution is a monotonically decreasing function of distance from the center of the sensor [7]. Da Rocha Gesualdi et al focused on the usage of the PCA in the recognition of character images. The main motivation for using principal components is to evaluate whether the relevant features for the classification task can still be extracted from a reduced number of components, which, in addition, may reduce the complexity of the neural classifiers Splitting the classification task into two, two expert neural classifiers were designed, one focusing on the identification of digits and another on letters. Both neural networks were trained with back propagation method and had a single hidden layer. This is specially designed for Brazilian license plate [8]. Mi-Ae Ko et al. proposed the method for recognizing is constructed from a feature-based approach, parameterized by an affine invariant parameters and the affine invariant features. Experimental results show that the performance of the proposed method is simple and robust, particularly when objects are heavily distorted with strong perspective view [9].

Fei Lu et al focused on extra dot appear in the license plate, this extra "dot" can completely affect the "shape descriptors", and confuse the recent character recognition methods. Solution propose by the author is first calculate the first a rough mean height is obtained, then pattern matching, with an XOR-based kernel, is used to find the precise bounding volume of the original character. Note that using the XOR-based kernel can improve the performance of the system drastically [10]. J1an-xia Wang et al. focused on the time complexity for template matching procedure. In this paper, license plate characters arrangement is as $\mathrm{X} 1 \mathrm{X} 2$ • $\mathrm{X} 3 \mathrm{X} 4 \mathrm{X} 5 \mathrm{X} 6 \mathrm{X} 7$, where $\mathrm{X} 1$ is Chinese characters, it is shortened forms of the Chinese provinces and cities, $\mathrm{X} 2$ is the English letter, it is the licensing authority department code, X3X4X5X6X7 is the combination of English letters and numbers. In order to enhance recognition speed and recognition rate, an improved template matching method is presented [11]. 


\section{FLOW DIAGRAM OF WORK}

A large and growing literature has been investigated and published on the Artificial intelligence and specially the Automatic vehicle number plate detection and vehicle number extraction. Automatic vehicle number plate detection and vehicle number extraction field and its application attracted many researchers to search and develop systems which can process images and get the useful information from them. This task is challenging due to the diversity of plate formats and the non uniform outdoor illumination conditions during image acquisition. Several techniques have been used to implement that job such as artificial neural network, template matching and fuzzy logic etc. This paper uses combination of both fuzzy logic and template matching to solve the problem of automatic vehicle number plate detection and vehicle number extraction [12]. We proposed a novel method to recognize vehicle number plate elements which are captured by any camera.

\section{METHODOLOGY}

The flow chart of the proposed system is shown in Fig.1.The first step is vehicle number plate detection which is followed by character and number segmentation, feature extraction \& recognition of the extracted feature and character recognition [12-14].

\section{A. Preprocessing(Vehicle number plate extraction)}

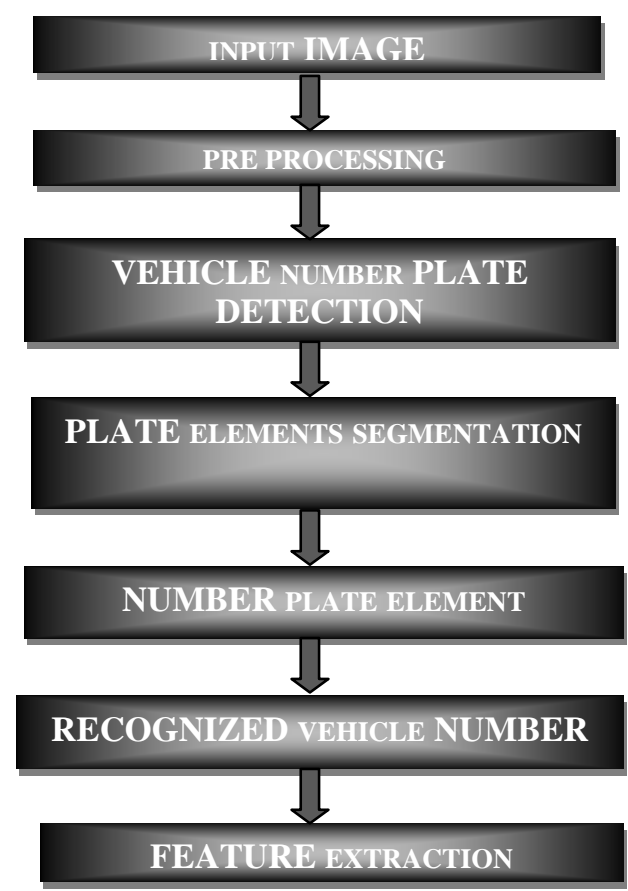

Fig. 1. Complete System Flowchart.

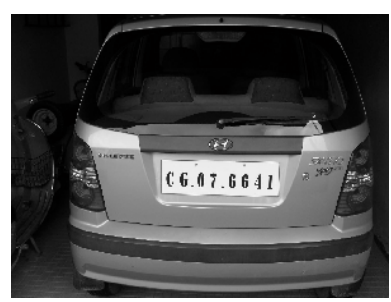

Fig 2. Captured Image.

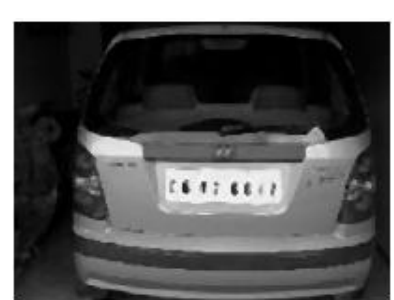

Fig. 3. Gray scale image.
In preprocessing we capture the image (Fig. 2) and convert it to gray image shown in Fig. 3. Fig. 4 shows the result of canny edge detection method and Fig. 5 depicts the output after vehicle number plate extraction.

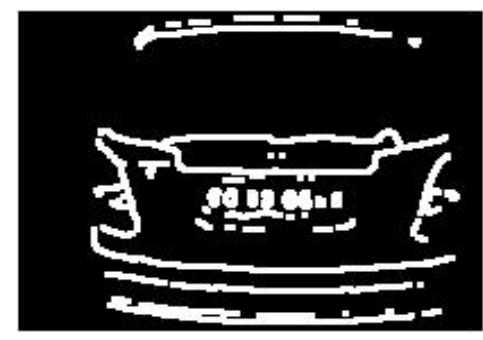

Fig. 4. Result of canny filter.

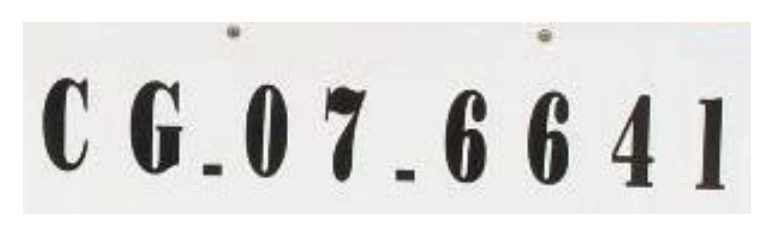

Fig. 5. Result of vehicle number plate extraction.

B. Vehicle Number Plate Elements Segmentation Preprocessing

Various preprocessing steps used are:

- Converting image to gray scale image- In photography and computing, a grayscale digital image is an image in which the value of each pixel is a single sample that is, it carries only intensity information. Images of this sort, also known as black-white, are composed exclusively of shades of gray, varying from black at the weakest intensity to white at the strongest. Image is converted to gray scale image.

- Converting image to black and white image - This image format also stores an image as a matrix but can only color a pixel black or white (and nothing in between). It assigns a 0 for black and a 1 for white.

- Removing all objects smaller than 100 pixels - In this step we use bwareaopen function to remove the small object which is present in number plate like dots of nut-bolt in the plate and other objects which is smaller then 100 pixels.

C. Removing extra area from left and right by detection of first and last columns for each character and number

The first step in segmentation process is to cutoff the background from each character and number from the license plate. We use vertical scanning to detect first and last columns for each character and number is conducted before horizontal scanning as explained in Algorithm .Vertical scanning is done before horizontal scanning because if skewness is present in the input image then its effect will be minimized. This improvement will reduce the error of first and last columns and rows which has been uncounted using our first approach. Vertical scanning (column by column) will be done to detect the first and last columns or each component and cut the area in between to separate the license information from background (shown in Fig. 6 and Fig. 7) 


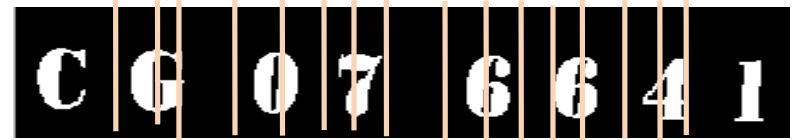

Fig. 6. Vertical Segmentation.

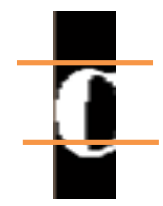

Fig. 7. Horizontal Segmentation.

\section{Role OF NeURAL NeTWORK AND FUZZY LOGIC}

In this paper we use fuzzy relations and Neural network for differentiating similar looking characters and then identifying correct alphabet [15-17]. First, we use fuzzy relations for identifying the similar looking alphabets i.e. similarity relation and then group them into single classes or in groups. Similarity relation means any fuzzy binary relation that is reflexive, symmetric and transitive, while equivalence relations clearly groups elements that are equivalent under the relation into disjoint classes. The similarity relation can be considered to effectively group elements into crisp sets whose members are "similar " to each other to some specified degree of parameter. When this degree is equal, the grouping is than an equivalence class. For each elements of $\mathrm{x}$ in $\mathrm{X}$, we can define a crisp set $A_{x}$, which contains all the elements of $X$ that are related to $x$ by the equivalence relation.: $\mathrm{A}_{\mathrm{x}}=\{\mathrm{y} \mid(\mathrm{x}, \mathrm{y}) \varepsilon \mathrm{R}(\mathrm{X}, \mathrm{X})\}, \mathrm{A}_{\mathrm{x}}$ is clearly a subset of $X$. The element $x$ is itself contained in $A_{x}$ due to the reflexivity of $R$; because $R$ is transitive and symmetric, each other member of $A_{x}$ is related to all the other members of $A_{x}$.Furthermore, no member of $A x$ is related to any element of $X$ is not included in $A_{x}$. This set $A_{x}$ is referred as an equivalence class of $R(X, X)$ with respect to $\mathrm{x}$.

Each similarity relation is associated with the set $\Pi(\mathrm{R})=$ $\left\{\Pi\left(\mathrm{R}_{\alpha}\right) \mid \alpha \varepsilon \Lambda_{\alpha}\right\}$ of partitions .Here $\alpha$ is a degree of similarity and $\mathrm{R}_{\alpha}$ is a $\alpha$-cut . In our methodology applied ,first we check the similarity relation with the help of Parameters (no. of holes ) and then we group them in to equivalence classes, like two hole class $(\mathrm{B}, 8)$, one hole class $(P, R, 9$ etc) and zero hole class $(G, C)$ and then again in this we pick the confusing characters from this equivalent classes and again check the similarity relation with some other parameter symbols like $\mid, /, 1$, _ like (X ,A , Y ) and then and according to similarity degree we put them into the equivalent classes. After applying fuzzy relations on alphabets we clearly get the equivalent classes and then using neural network technique for identifying the exact character on training set of data [16-17] . A neural network $(\mathrm{NN})$ is an artificial network model, which emulates the cerebral nerve network in the brain [18]. A typical NN is a Multilayer feed forward neural network trained by the popular back-propagation method [13]. The goal of the training process is to find the set of weight values that will cause the output from the neural network to match the actual target values as closely as possible. All neural networks have an input layer and an output layer, but the number of hidden layers may vary as per requirement. When character domain could be obtained, we can perform character recognition in high accuracy by a neural network. Fig. 8 shows Multilayer feed forward neural network with two hidden layers and four total layers:

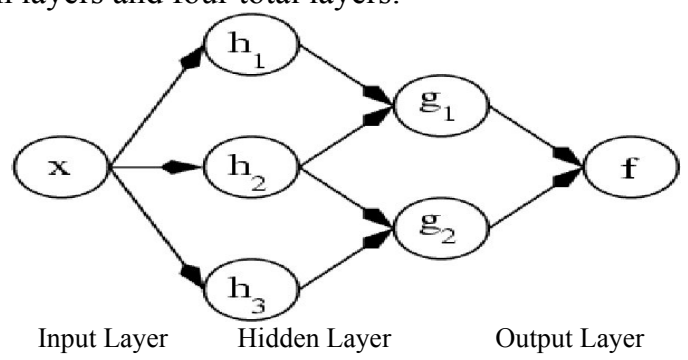

Fig. 8. Multilayer Feed Forward Neural Network.

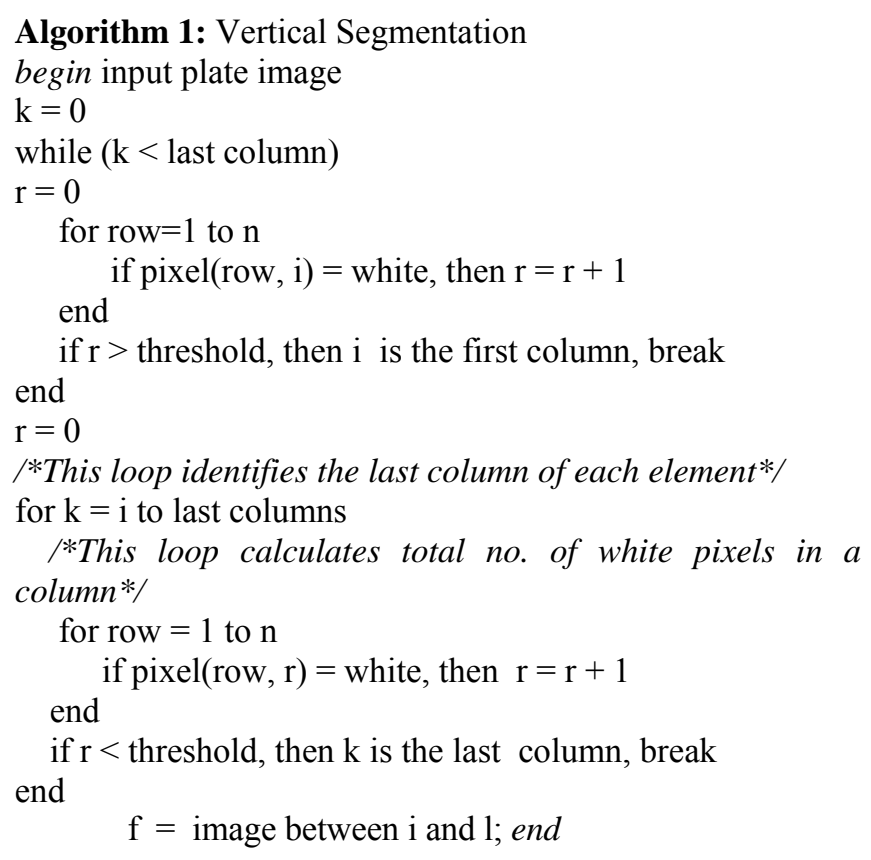

Algorithm 2: Horizontal Segmentation

begin input $\mathrm{f}$

$\mathrm{m}=0 \mathrm{n}=0$

for $\mathrm{t}=$ first row to last row

if $\operatorname{pixel}(\mathrm{t})=$ white, then $\mathrm{m}=\mathrm{m}+1$

end

if $\mathrm{m}>$ threshold, then $\mathrm{i}$ is the first row, break end

/*This loop identifies the ending row of each element*/

for $\mathrm{t}=$ first row to last row

/*This loop calculates total no. of white pixels in a column*/

for $\mathrm{r}=\mathrm{i}$ to last row

if $\operatorname{pixel}(r)=$ white then $n=n+1$

end

if $\mathrm{n}<$ threshold, then 1 is last row, break

end

$\mathrm{d}=$ image between $\mathrm{i}$ and $\mathrm{l}$; end

\section{A. Post Processing : Feature extraction}

After the first and last columns of the component have been detected, horizontal scanning will be done to detect the first and last rows from the result of the previous step and 
the whole process is described in Algorithm 2. This result will help to extract image features easily. Grouping the numbers and characters on the basis of holes (Euler Number)- First step is to calculate the no of holes for each character and number image and give them some number 1 , $0 .-1$. This number is calculated, the number of connected elements (always equal to one) minus the number of holes. Three groups will result from this step as follows:

- Number equal to 1 - This group contains C, E, F, G, H, I, J, K, L, M, N, S, T, U, V, W, X, Y and Z. It also contains the numbers $1,2,3,5$ and 7 .

- Number equal to 0 - This group contains A, D, O, P, $\mathrm{Q}$ and $\mathrm{R}$ and it contains number 4,6 and 9.

- Number equal to -1 - This group only contains character $\mathrm{B}$ and number 8 .

It is clear that this grouping process will increase system accuracy of recognizing characters and numbers and it will also reduce the time of recognition process [12]. For $\mathbf{E}$, the number of holes is 0 , so the Euler number can be calculated as:

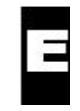

Euler no. of $E=1-$ no. of hole $=1-0=1$

For A, the number of holes is 1, so the Euler number can be calculated as:

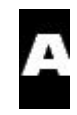

Euler no. of $A=1-$ no. of hole $=1-1=0$

For $\mathrm{B}$, the number of holes is 2, so the Euler number can be calculated as:

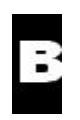

Euler no. of $\mathrm{B}=1-$ no. of hole $=1-2=-1$

This process continues for all other characters and different Euler groups are classified [10-12].

\section{B. Character and number recognition}

Here we use a new methodology in which the characters are separated into subgroups on the basis of their similarity or different physical appearance. Euler group 1 is divided into the following subgroups

- Subgroup C: consists of C and G;

- $\quad$ Subgroup E: Consists of E and F;

- $\quad$ Subgroup K: Consists of K, X and Y;

- $\quad$ Subgroup I: Consists of I, T, J and 1;

- Subgroup S: Consists of 5 and S.

Euler group 2 is divided into the following subgroups:

- $\quad$ Subgroup O: consists of O and Q;

- $\quad$ Subgroup P : consists of P and R.

Remaining elements are not categorized into any subgroup and have a separate template for each of them.

Each group has a template; the template that matches with the input the most decides the group of the character. The subgroup is decided on the basis of indexes calculated by formulae:

Index of a particular template $=$

(Number of pixels that matched with that template) / (Total number of pixels in that template)

The subgroup whose template has highest index with respect to the character is chosen as the subgroup. Once, we get the subgroup then the physical characteristics of each element that differentiate it from other elements are used to identify the element. For example: In Subgroup E: E and F are members of this subgroup. The template shown in Fig. 9 is used to check, if the input belongs to this group then the template shown in Fig. 10 is used to detect if the input is E or $\mathrm{F}$ as this template is a part of $\mathrm{E}$ but not $\mathrm{F}$.

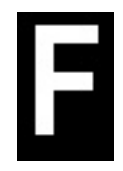

Fig. 9. Template to judge subgroup.

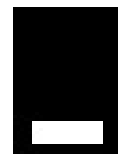

Fig. 10. Template to judge group whether $\mathrm{E}$ or $\mathrm{F}$.

The total number of clustring of pixels in the upper half of $\mathrm{I}$ is almost equal to that of lower half,but in the case of $\mathrm{T}$ is $60 \%$ more than that in the lower half,this feature is used to differentiate betweem I \& T.Thus, the overall,efficiency of the system is increased by implementing this fuzzy logic. Another algorithm for post processing for detection of characters and numbers is as under:

$\mathrm{c}=1 / / \mathrm{c}$ is the number of connected components (always equal to one); $\mathrm{h}=$ number of holes in character $\mathrm{e}=\mathrm{c}-\mathrm{h}$

If $\mathrm{e}=1$, then $\mathrm{eg}=1$

Else if $\mathrm{e}=0$ then $\mathrm{eg}=2$

Else if $\mathrm{e}=-1$ then $\mathrm{eg}=3$

End if

For all elements, Index $[\mathrm{i}]=0 / /$ Index of a particular template if $\mathrm{eg}=1$ then //it is divided into subgroups further for all elements of $\mathrm{eg}=1$ eg1_index[]=element index(template t[]) maximum (eg1_index)

$\mathrm{x}=$ position of template where maximum matched if $\mathrm{x}=3$ then , subgroup $=\mathrm{C}$

sbg1_index[]=element index(template $\mathrm{t}[])$ maximum (sbg1_index)

//match with respective subgroup templates like C,G for subgroup C

For obtained $\mathrm{x}$; Return character(x)

\section{RESUlT AND DisCUSSION}

The evaluation of the proposed technique, when the bipartitioned images are given to the algorithm proposed the characters have been recognized. The results are shown in the Table I. We have proposed a new method for character recognition which very effectively eliminates the problem of confusion between characters of same type like $\mathrm{C} \& \mathrm{G}$ which the previous algorithms were not able to do with high precision. Initial experiments have shown success in 
differentiating between similar looking characters. The above mentioned method inspite of being very effective on standard number plate suffers with the disadvantage that it is not usefull on those plate which use non-standard characters to represent the vehicle's registration number. When evaluating with different license plate characters, the technique has recognized the characters with an accuracy rate of $95 \%$.

TABLE I. RECOGNIZED CHARACTERS.

\begin{tabular}{|c|c|c|c|c|c|}
\hline SN & 1 & 2 & 3 & 4 & 5 \\
\hline Suljett & & & & & \\
\hline Berults & $\mathbf{M}$ & $\mathrm{H}$ & 0 & 2 & G \\
\hline
\end{tabular}

\section{CONCLUSIONS}

The achieved accuracy rate is remarkable and will make the technique to apply in the concerned applications. Ways to implement the above mentioned character recognition algorithm on non-standard vehicle number plates are still to be found. Also,the above mentioned algorithm doesnot gives optimum result when skewness is present in the detected vehicle number plate, the efficiency of the vehicle number plate detection algorithm used in this paper limits the efficiency of the total system.

\section{REFERENCES}

[1] G. Serkan Ozbay and Ergun Ercelebi, "Automatic Vehicle Identification by Plate Recognition", World Academy of Science, Engineering and Technology, Vol.9, No.41, pp.222-225, 2005.

[2] I. Broumandnia and Fathy, "Application of pattern recognition for Farsi license plate recognition", ICGST International Journal on Graphics, Vision and Image Processing, Vol.5, No.2, pp.25-31, Jan 2005.

[3] K Hsien-Chu WU, Chwei-Shyong TSAI and Ching-Hao LAI, "A License Plate Recognition System in E-Government", International Journal of Information and Security, Vol.15, No.2, pp.199-210, 2004.

[4] R Sorin Draghici, "A neural network based artificial vision system for licence plate recognition", International Journal of Network Security, International Journal of Neural Systems, Vol.8, No.1, pp.113-126, 1997.

[5] Y. I-Chen Tsai, Jui-Chen Wu, Jun-Wei Hsieh and Yung-Sheng Chen, "Recognition of Vehicle License Plates from a Video Sequence", IAENG International Journal of Computer Science, Vol.36, No.1, pp.26-33, February 2009.

[6] M. Kaushik Deb, Hyun-Uk Chae and Kang-Hyun Jo, "Vehicle License Plate Detection Method Based on Sliding Concentric Windows and Histogram", Journal of Computers, Vol. 4, No. 8, pp.771-777, August 2009.

[7] E Ping -Wen Ong, Richard S. Wallace, Eric L. Schwartz "SpaceVariant Optical Character Recognition”. Pattern Recognition, 1992. Vol. II. Conference B: Pattern Recognition Methodology and Systems, Proceedings. 11th IAPR International Conference on 30 Aug 1992 03 Sep 1992.

[8] Aline da Rocha Gesualdi, José Manoel de Seixas, Marcelo Portes de Albuquerque, Márcio Portes de Albuquerque, "Character Recognition in Car License Plates Based on Principal Components and Neural Processing," Neural Networks, Brazilian Symposium on, pp. 206, VII Brazilian Symposium on Neural Networks (SBRN'02), 2002.

[9] H Mi-Ae Ko, Young-Mo Kim, "A simple OCR method from strong perspective view" ,Proceedings of the 33rd Applied Imagery Pattern
Recognition Workshop (AIPR'04) South Korea , 13-15 Oct. 2004 On page(s): 235 - 240

[10] Fei Lu Mei Xie , "An Efficient Method of License Plate Location in Complex Scene”, ICCMS '10, Volume: 2, pp. 206 - 209, 22-24 Jan., 2010.

[11] Jian-Xia Wang, Wan-Zhen Zhou and Xin Liu, "The research and realization of vehicle license plate character segmentation and recognition technology" , Proceedings of the 2010 International Conference on Wavelet Analysis and Pattern Recognition, Qingdao, 11-14 July 2010

[12] Wisam Al-faqheri and Syamsiah Mashohor, "Real-Time Malaysian Automatic License Plate Recognition (M-ALPR) using Hybrid Fuzzy", IJCSNS International Journal of Computer Science and Network Security, Vol. .9, No.2, February 2009.

[13] J . Hearts , A. Krogh, Richard and G. Palmer, Introduction to The Theory of Neural Compution, Addison-Wesley Publishing Company, The Advance Book Program , 350 Bridge Park0way, Redwood City, 1991.

[14] Zhai, H.C., Chavel, P., Wang, Y., "Weighted fuzzy correlation for similarity measure of color-histograms.", Optics Comm. 247, 49-55, 2005.

[15] Y.K.Siah, T.Y.Haur, M.Khalid, and T.Ahmad "Vehicle License Plate Recognition by Fuzzy ARTMAP Neural Network", Proc.of WEC'99, pp 19-22, 1999.

[16] M. Alata and M. A. Shabi, "Text detection andcharacters recognition using fuzzy image processing,"Journal of Electrical Engineering, vol. 57, no. 5, pp.258-267, 2006.

[17] J. A. G. Nijhuis, M. H. T. Brugge, K. A. Helmholt, J.P. W. Pluim, L. Spaanenburg, R. S. Venema, and M.A. Westenberg, "Car license plate reconition with neural networks and fuzzy logic," in IEEE Int.Conference on Neural Networks, vol. 5, pp. 2232-2236, 1995.

[18] M. Fukumi and Y. Takeuchi, "Neural network based threshold determination of malaysian license plate character recognition," in Intelligent Signal Processing and Communication Systems, pp.771775,2004 .

Choubey Siddhartha born on $25^{\text {th }}$ May 1976 in India. He obtained his Bachelar of Engineering in Computer Science \& Engineering and M.Tech. in Computer Technology \& Applications (2008) from Chhattisgarh Swami Vivekanand Technical University (CSVTU) Bhilai, India. He is pursuing $\mathrm{Ph} . \mathrm{D}$. in Computer Science \& Engineering from MATS University, Raipur, India. His research interest includes digital image processing, biomedical imaging and neuro-fuzzy based pattern recognition.

He is working as Associate Professor (Computer Science \& Engineering) in Shri Shankaracharya College of Engineering and Technology, Bhilai, India. He has 8 years of teaching experience.

Sinha G.R. born in India on $4^{\text {th }}$ March 1975. He obtained his Bachelor of Engineering in Electronics (1998) and M.Tech. in Computer Technology (2004) with Gold Medal from National Institute of Technology, Raipur, India. He obtained Ph.D. in Electronics \& Telecommunication Engineering (2010) from Chhattisgarh Swami Vivekanand Technical University (CSVTU) Bhilai, India. His field of interest includes digit image processing $\&$ its applications, early detection of breast cancer, license plate recognition, nano imaging, and underwater imaging.

$\mathrm{He}$ is working as Professor (Electronics \& Telecommunication Engineering) \& Head (Information Technology) in Shri Shankaracharya College of Engineering and Technology, Bhilai, India. He has 13 years of experience in teaching \& research and has published more than 92 research papers in various International and National journals and conferences. He is active reviewer and editorial board member of more than 12 International journals.

Dr. Sinha is member of IEEE, IACSIT and many national professional organizations such IETE, ISTE, IEI, CSI, ISCA, IJERIA. He is recipient of many awards such as Engineer of the year award 2008, Expert Engineer award 2007, CG Young Scientist award 2005, Nominated for ISCA Indian Young Scientist award and Deshbandhu merit scholarship.

Patel Bhagwati Charan born on $5^{\text {th }}$ July 1979 in India. He obtained his M.Tech. in Information Technology (2005) from Rajiv Gandhi Technical University, Bhopal, India. His research interest includes digital image processing, biomedical imaging and neuro-fuzzy based pattern recognition.

$\mathrm{He}$ is working as Associate professor (Information Technology) in Shri Shankaracharya College of Engineering and Technology, Bhilai, India. He has 6 years of teaching experience.

Choubey Abha born on $6^{\text {th }}$ October 1983 in India. She obtained her Bachelar of Engineering in Computer Science \& Engineering and M.Tech. 
in Computer Technology \& Applications (2008) from Chhattisgarh Swami Vivekanand Technical University (CSVTU) Bhilai, India. She is pursuing $\mathrm{Ph} . \mathrm{D}$. in Computer Science \& Engineering from MATS University, Raipur, India. Her research interest includes digital image processing and biomedical imaging.

She is working as Associate Professor (Computer Science \& Engineering) in Shri Shankaracharya College of Engineering and Technology, Bhilai, India. He has 7 years of teaching experience.

Thakur Kavita born on $7^{\text {th }}$ December 1967 in India. She obtained Master of Engineering in Electronics \& Telecommunication Engineering (1991) from University of Roorkee and Ph.D. from Pt. Ravishankar Shukla University, Raipur, India. Her research interest includes speech processing, image processing and microcontroller based applications.

She is working as Reader in School of Electronics of Pt. Ravishankar Shukla University, Raipur, India. She has 17 years of experience in teaching and research.

Dr. Thakur is member of IEEE, IEI, CSI and ASI. 